\title{
Oblique shock reflection from an axis of symmetry: shock dynamics and relation to the Guderley singularity
}

\author{
By H. G. HORNUNG ${ }^{1}$ AND D. W. SCHWENDEMAN \\ ${ }^{1}$ Graduate Aeronautical Laboratories, California Institute of Technology, \\ Pasadena, CA 91125, USA \\ ${ }^{2}$ Department of Mathematical Sciences, Rensselaer Polytechnic Institute, Troy, NY 12180, USA
}

(Received 31 July 2000 and in revised form 5 December 2000)

Oblique shock reflection from an axis of symmetry is studied using Whitham's theory of geometrical shock dynamics, and the results are compared with previous numerical simulations of the phenomenon by Hornung (2000). The shock shapes (for strong and weak shocks), and the location of the shock-shock (for strong shocks), are in good agreement with the numerical results, though the detail of the shock reflection structure is, of course, not resolved by shock dynamics. A guess at a mathematical form of the shock shape based on an analogy with the Guderley singularity in cylindrical shock implosion, in the form of a generalized hyperbola, fits the shock shape very well. The smooth variation of the exponent in this equation with initial shock angle from the Guderley value at zero to 0.5 at $90^{\circ}$ supports the analogy. Finally, steady-flow shock reflection from a symmetry axis is related to the self-similar flow.

\section{Introduction}

This study is concerned with the reflection of an inward-facing, initially conical shock wave from its axis of symmetry. The flow is considered to be inviscid, and the medium is taken to be a perfect gas. An exploratory computational study of this problem (Hornung 2000), based on numerical solutions of the Euler equations, discovered that the convergence of space toward the axis of symmetry causes such flows to differ from corresponding plane flows in several ways:

the shock steepens as it approaches the axis, in a manner analogous to the Guderley singularity (see Whitham 1974) in the implosion of a cylindrical shock;

no regular reflection can occur;

for strong shocks, a vortex ring follows the Mach stem and deforms it, so that two new Mach reflection configurations occur.

The exploratory study related numerical results for conical shock reflection to features observed in shock reflection in a shock tunnel and in the diffraction of a shock over a heavy spherical bubble or a solid sphere. In the case of shock reflection in a shock tunnel and shock diffraction over a heavy spherical bubble, a vortex ring is formed which, for strong shocks, overtakes and deforms the Mach disk, creating complex Mach reflection configurations. Depending on the degree of interaction, these configurations were labelled (DV) for bulging Mach reflection with two triple points or (V) for vortical Mach reflection in which the vortex ring dominates the Mach disk (Hornung 2000). In the case of weaker shocks, the vortex ring and Mach disk do not 
interact and a simple Mach reflection (S) is observed. Planar shock diffraction by a solid sphere was studied both theoretically and experimentally by Bryson \& Gross (1961) for incident shock Mach numbers equal to 2.85 and 4.41. Many of the features observed for this problem may be seen in the conical Mach reflection problem at low and intermediate shock strengths, where both the (S) and (DV) configurations are observed, although the latter is only faintly discernible.

Using Whitham's theory of geometrical shock dynamics (Whitham 1974), it is possible to compute and study the behaviour of the leading shock fronts for the problem of conical Mach reflection. The initial conical shock is described by its initial Mach number $M_{\mathrm{s}}$ and cone angle $\beta$. There is no length scale and the resulting flow is assumed to be axisymmetric. In this case, the flow is self-similar so that the equations of geometrical shock dynamics reduce to ordinary differential equations whose solution describes the shock Mach number and inclination angle of the shock along the shock front. This solution is connected to the Mach disk via jump conditions across a shock-shock (corresponding to the location of the triple-point in the full theory of gas dynamics). Whitham's theory is limited to the description of the leading shock front and thus complex Mach reflection configurations such as (DV) and (V), which result from flow features that form behind the lead shock and overtake it, are not fully described.

The solution construction for conical Mach reflection within the theory of shock dynamics is similar to that for planar shock diffraction by a cone (Whitham 1959). The same similarity form appears, but for shock diffraction by a cone the equations are solved for the curved, annular Mach stem on the opposite side of the shock-shock. Bryson \& Gross (1961) found good agreement between the shock-shock positions computed for this problem and triple-point positions observed experimentally.

Both the numerical calculations of the Euler equations discussed in the exploratory study and the shock dynamics calculations to be presented here impose axial symmetry. They are therefore not able to detect three-dimensional phenomena that might arise from instability of the shock, presumably near the edge of the Mach disk. In fact, the cylindrical shock implosion is known to be unstable (see Whitham 1974, and for experiments see e.g. Takayama, Klein \& Grönig 1987). However, one may expect the oblique implosion studied here to be more stable, since a Mach stem inevitably forms at the axis (see Rylov 1990). Nevertheless, we cannot rule out the occurrence of three-dimensional instability. Converging shocks were re-examined using shock dynamics by Schwendeman \& Whitham (1987) and by Schwendeman (1993). These studies revealed that the formation of Mach stems in the converging shocks led to ultimate stability.

Using the results of the computations made in the exploratory study, the aims here are: to investigate the shock steepening quantitatively, and to test the numerical solutions by using Whitham's shock dynamics theory; to investigate the relation of these flows to the Guderley solution in a quantitative fashion; and to explore shock reflection from a symmetry axis in steady flow.

\section{Problem parameters and numerical method of solution}

The flow is assumed to be inviscid and axisymmetric, and the medium is taken to be a perfect gas. The results of the exploratory study, which will be used here, were obtained by numerical solution of the unsteady Euler equations. The computation is started from the following initial condition: along a conical surface a pressure and temperature discontinuity is specified, such that the pressure on the inside of the cone 
is lower than that on the outside. When the flow evolves from this state, a shock of initially uniform strength propagates toward the cone axis, followed by a contact surface, and an expansion wave propagates outward. The initial shock Mach number, $M_{\mathrm{s}}$, depends on the initial pressure and temperature ratios, $p_{4} / p_{1}, T_{4} / T_{1}$. Thus, the parameters controlling this phenomenon are these two ratios, the specific heat ratio of the gas, $\gamma$, and the angle of the conical discontinuity, $\beta$. Even though the solution depends on $x / t$ and $r / t$ only, it is simpler to compute solutions of the unsteady equations and then present results at an arbitrary fixed time.

Numerical solutions of this problem are given in the exploratory study, and these solutions will be used here. These Euler computations were performed using the software system Amrita, constructed by James Quirk, see Quirk (1998). Amrita is a system that automates and packages computational tasks in such a way that the packages can be combined (dynamically linked) according to instructions written in a high-level scripting language. In this application, features of Amrita were used that include the automatic construction of different Euler solvers, automatic documentation of the codes, automatic adaptive mesh refinement according to simply chosen criteria, and scripting-language-driven computation, archiving and post-processing of the results. The Euler solver generated for the computations was an operatorsplit scheme with HLLE flux and kappa-MUSCL reconstruction. Adaptive mesh refinement was applied to a $200 \times 120$ mesh in two tiers of a factor of 3 , so that the effective mesh was $1800 \times 1080$. Because the problem is self-similar (growing linearly in time) a very effective mesh refinement sensitivity study can be made by computing for a long time. The early times then correspond to a coarse grid, while the structures are much better resolved at later times. This is demonstrated by the self-similarity test of Hornung (2000), which revealed the fine-detail accuracy of the computations as well as the parameter range where mesh problems arise. In particular, well-understood problems are encountered in the region of very small incident shock angles $\left(\beta<20^{\circ}\right)$. However, it is the larger shock angles that give the more interesting flows.

\section{Incident shock steepening}

Consider that portion of an axisymmetric flow which lies between two radial planes through the axis. These planes will be flow planes, i.e. the velocity has no normal component to them. If the velocity has a component normal to the axis and inward, then the constraint imposed by these two flow planes causes streamlines to converge laterally. In steady, supersonic flow this would cause the pressure to increase. Thus, an oblique inward-facing conical shock will steepen near the symmetry axis. This has been observed by Mölder et al. (1997). We shall return to some examples of the steady-flow case in $\S 6$.

In the pseudosteady case considered here, the lateral convergence has the same effect of increasing the pressure and strengthening the shock as it approaches the axis. The closer the shock gets to the axis, the faster this lateral squeezing effect becomes, and consequently the more rapidly the shock steepens. The intersection of the shock with the symmetry axis has to occur at right angles. However, when the shock angle exceeds the von Neumann angle, or thereabouts, transition to Mach reflection has to occur, so that regular reflection is not possible.

As indicated in the exploratory study, the numerical solution of this problem is subject to the difficulty that the solution in the vicinity of the axis is suspect. This is because any singular behaviour cannot be resolved with a discrete mesh, so that features observed could be artifacts of the discretization. There is always 
some concern about numerical computations of the Euler equations, particularly when the effects of discretization fail to permit resolution of singular regions. It is therefore interesting to compare these numerical results with corresponding results using Whitham's approximate theory of geometrical shock dynamics. Good agreement is expected for strong shocks, but it is also interesting to compare the results for a wide range of initial shock strengths including weak shocks.

\section{Shock dynamics}

The equations of geometrical shock dynamics are

$$
\nabla \cdot\left(\frac{M}{A} \nabla \alpha\right)=0, \quad M=\frac{1}{|\nabla \alpha|}, \quad A=A(M),
$$

where the shock front position at a time $t$ is given by the surface $\alpha(x, y, z)=a_{0} t$ ( $a_{0}$ being an ambient sound speed), $M$ is the Mach number of the shock, and $A(M)$ is an assumed functional relationship between $M$ and an area of the ray tube $A$ (cf. Whitham 1974, equations 8.50-8.52). Following Whitham (1959), the secondorder partial differential equation (PDE) in (4.1) may be written as a system of two first-order PDEs for the case of axisymmetric flow. Let

$$
\frac{\partial \alpha}{\partial x}=\frac{\cos \theta}{M} \text { and } \frac{\partial \alpha}{\partial r}=\frac{\sin \theta}{M},
$$

where $r^{2}=y^{2}+z^{2}$ and $\theta$ is the angle between the shock normal and the axis of symmetry. By definition

$$
\frac{\partial}{\partial x}\left(\frac{\sin \theta}{M}\right)-\frac{\partial}{\partial r}\left(\frac{\cos \theta}{M}\right)=0
$$

and from (4.1) we have

$$
\frac{\partial}{\partial x}\left(\frac{r \cos \theta}{A}\right)+\frac{\partial}{\partial r}\left(\frac{r \sin \theta}{A}\right)=0 .
$$

For the problem of conical Mach reflection, the solution depends on the initial shock Mach number $M_{\mathrm{s}}$ and angle of discontinuity $\beta$ only (assuming a fixed specific heat ratio $\gamma$ ). There is no length scale, as mentioned previously. Accordingly, $M$ and $\theta$ are functions of the similarity variable $\eta=\arctan (r / x)$ and thus (4.2) and (4.3) become

$$
\frac{1}{M} \frac{\mathrm{d} M}{\mathrm{~d} \eta}=\tan (\eta-\theta) \frac{\mathrm{d} \theta}{\mathrm{d} \eta}
$$

and

$$
\tan (\eta-\theta)\left(-\frac{1}{A} \frac{\mathrm{d} A}{\mathrm{~d} M}\right) \frac{\mathrm{d} M}{\mathrm{~d} \eta}=\frac{\mathrm{d} \theta}{\mathrm{d} \eta}+\frac{\tan \theta}{\sin \eta \cos \eta(1+\tan \eta \tan \theta)},
$$

respectively. We seek a solution to (4.4) and (4.5) for $\eta$ between the angles $\chi$ and $\beta$ subject to various boundary conditions as illustrated in figure 1 . As $\eta$ approaches the initial angle of discontinuity $\beta, M$ and $\theta$ approach $M_{\mathrm{s}}$ and $\beta-\pi / 2$, respectively. At $\eta=\chi, M=M_{1}$ and $\theta=\theta_{1}$, where the values for $M_{1}$ and $\theta_{1}$ must be connected to the values $M_{2}$ and $\theta_{2}=0$ for the Mach stem by the so-called shock-shock relations (cf. 


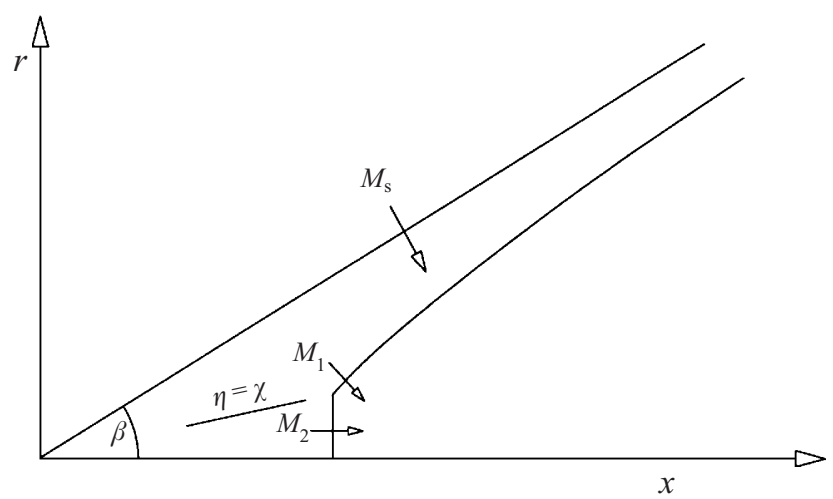

FIGURE 1. Coordinate system for shock dynamics, showing initial shock location, strength $M_{\mathrm{s}}$ and later shock location with shock jump from strength $M_{1}$ to $M_{2}$ across the shock-shock.

Whitham 1974, equations 8.81-8.82). These equations provide the boundary conditions

$$
\left.\begin{array}{rl}
-\tan \theta_{1} & =\frac{\left(M_{2}^{2}-M_{1}^{2}\right)^{1 / 2}\left(A_{1}^{2}-A_{2}^{2}\right)^{1 / 2}}{A_{2} M_{2}+A_{1} M_{1}} \\
\tan \chi & =\frac{A_{2}}{M_{2}}\left(\frac{M_{2}^{2}-M_{1}^{2}}{A_{1}^{2}-A_{2}^{2}}\right)^{1 / 2}
\end{array}\right\} \quad \text { at } \eta=\chi,
$$

where $A_{1}=A\left(M_{1}\right)$ and $A_{2}=A\left(M_{2}\right)$. As noted previously, the problem formulation is close to that for shock diffraction by a cone (Whitham 1959) except that the disturbed region is on the opposite side of the shock-shock.

A numerical method is needed to determine the similarity solution. The basic procedure we use starts with a provisional choice for $M_{1}$ and $M_{2}$ which in turn gives values for $\theta_{1}$ and $\chi$ from (4.6). Then, the system of ordinary differential equations (ODEs) given by (4.4) and (4.5) is integrated numerically for increasing $\eta$ using $M=M_{1}$ and $\theta=\theta_{1}$ at $\eta=\chi$. As $\eta$ increases, $\eta-\theta$ approaches $\pi / 2$ and we find values for $M$ and $\theta$ which do not approach the desired values of $M_{\mathrm{s}}$ and $\beta-\pi / 2$ in general. A method of iteration (Newton's method) is used to adjust the values of $M_{1}$ and $M_{2}$ until the boundary conditions at $\eta=\beta$ are satisfied.

For the case of a strong shock in which $M_{\mathrm{s}} \gg 1$, the area-Mach number relation suggested by Whitham reduces to

$$
A=M^{-n}, \quad n=5.0743 \text { for } \gamma=1.4,
$$

which simplifies the calculation. In this case,

$$
-\frac{M}{A} \frac{\mathrm{d} A}{\mathrm{~d} M}=n
$$

and (4.4) and (4.5) reduce to a single equation for $\theta$, namely

$$
\left(n \tan ^{2}(\eta-\theta)-1\right) \frac{\mathrm{d} \theta}{\mathrm{d} \eta}=\frac{\tan \theta}{\sin \eta \cos \eta(1+\tan \eta \tan \theta)} .
$$

This equation is to be solved subject to the boundary conditions

$$
\theta \rightarrow \beta-\pi / 2 \text { as } \eta \rightarrow \beta
$$




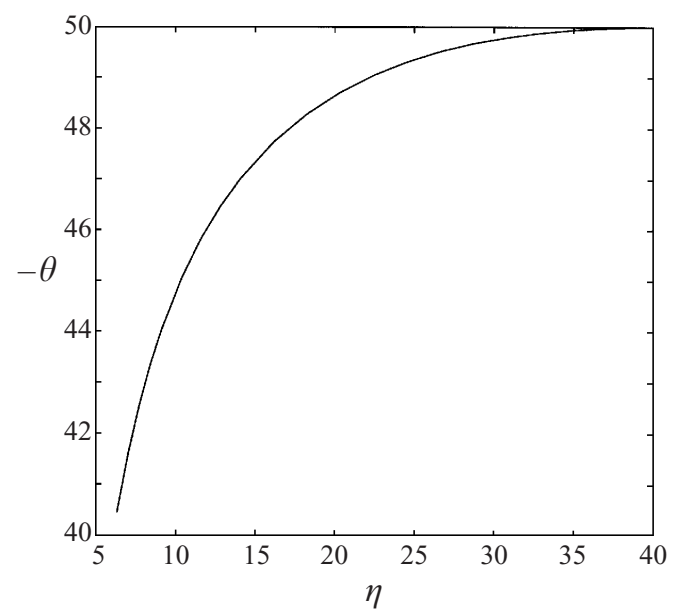

FIGURE 2. Similarity solution in the strong shock limit for $\beta=40^{\circ}$ and $\gamma=1.4$. (All angles are given in degrees.)

and

$$
\left.\begin{array}{rl}
-\tan \theta_{1} & =\frac{\left(1-\sigma^{2}\right)^{1 / 2}\left(\sigma^{-2 n}-1\right)^{1 / 2}}{1+\sigma^{1-n}} \\
\tan \chi & =\left(\frac{1-\sigma^{2}}{\sigma^{-2 n}-1}\right)^{1 / 2}
\end{array}\right\} \text { at } \eta=\chi,
$$

where $\sigma=M_{1} / M_{2}<1$. For this case, a choice for $\sigma$ is made which leads to values for $\theta_{1}$ and $\chi$ from (4.9). Then, (4.8) may be integrated numerically from $\eta=\chi$ to $\eta-\theta=\pi / 2$. The value for $\sigma$ is adjusted until $\theta=\beta-\pi / 2$ when $\eta=\beta$. Once a solution for $\theta$ is found, the shock Mach numbers may be found by integrating (4.4) using $M_{1}=1$, which chooses a normalization of the shock Mach number in the strong shock limit.

The positions of the shock fronts may be found from the solutions for $M$ and $\theta$. The similarity form for $\alpha$ is $x f(\eta)$ so that

$$
\frac{\partial \alpha}{\partial x}=f-\left(\frac{\tan \eta}{1+\tan ^{2} \eta}\right) f^{\prime}=\frac{\cos \theta}{M}
$$

and

$$
\frac{\partial \alpha}{\partial r}=\left(\frac{1}{1+\tan ^{2} \eta}\right) f^{\prime}=\frac{\sin \theta}{M} .
$$

We may now eliminate $f^{\prime}$ from (4.10) and (4.11) and use $\alpha=x f(\eta)=a_{0} t$ to give

$$
\frac{x}{a_{0} t}=\frac{M}{\cos \theta(1+\tan \eta \tan \theta)} \text { and } \quad \frac{r}{a_{0} t}=\frac{x}{a_{0} t} \tan \eta \text {. }
$$

Following the numerical procedures outlined above, solutions have been computed using the full area-Mach number relation and using the reduced form in the strong shock limit. Figure 2 shows the behaviour of $\theta$ as a function of $\eta$ in the strong shock limit when $\beta=40^{\circ}$. The value of the shock-shock angle, $\chi$, for this case is $6.324^{\circ}$. The variation in $\theta$ is greatest for smaller values of $\eta$ corresponding to shock positions near the axis of symmetry, as expected. The overall variation in $\theta$ is not large, only 

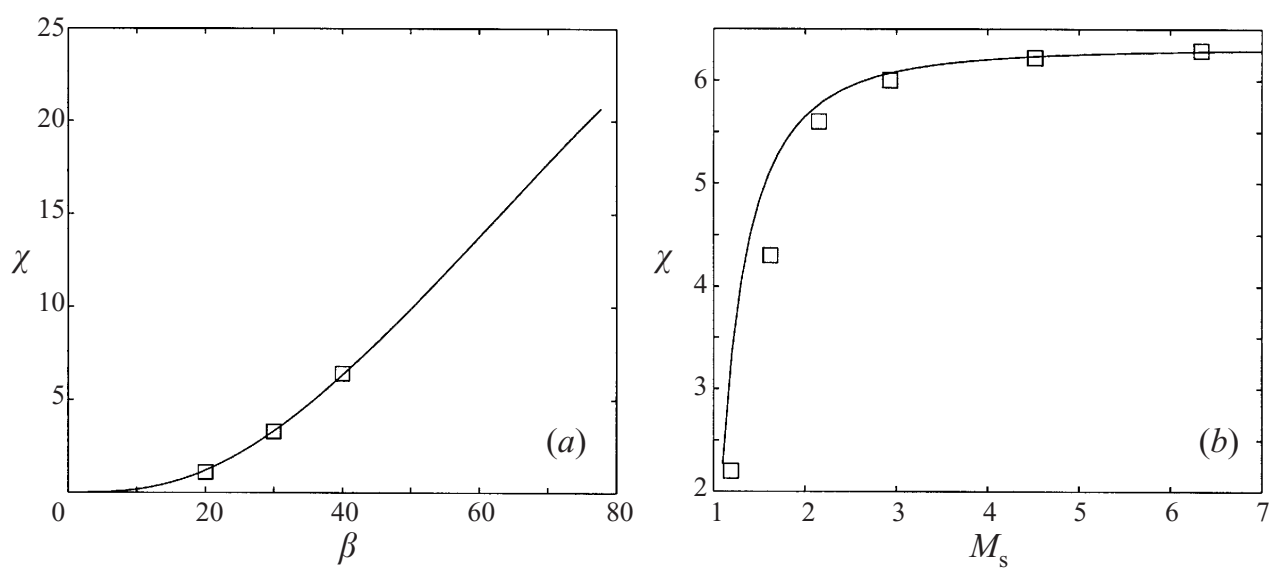

FIGURE 3. Behaviour of the shock-shock angle, $\chi,(a)$ as a function of $\beta$ in the strong shock limit and $(b)$ as a function of $M_{\mathrm{s}}$ using the full area-Mach number relation and $\beta=40^{\circ}$. Symbols are triple-point locations from corresponding Euler calculations $\left(M_{\mathrm{s}}=6.34\right.$ is used in $(a)$ for the strong shock case).

about $10^{\circ}$ for this case, indicating that there is not a large deviation from the case of planar Mach reflection. The behaviour of the shock-shock angle $\chi$ as a function of $\beta$ is shown in figure $3(a)$ in the strong shock limit and its behaviour as a function of $M_{\mathrm{s}}$ is shown in figure $3(b)$ using the full area-Mach number relation when $\beta=40^{\circ}$. Shock-shocks in Whitham's approximate theory correspond to the location of triple points. For comparison, we have computed corresponding solutions of the unsteady Euler equations and determined triple-point locations from the results which in turn are marked in figure 3. For the strong shock case, figure 3(a), the incident shock Mach number is $M_{\mathrm{s}}=6.34$ and we note excellent agreement with the shock-shock angle from geometrical shock dynamics, while for weaker initial shocks, figure $3(b)$, we observe some deviation.

Positions of the shock fronts given by shock dynamics may be calculated and compared with solutions of the unsteady Euler equations at any given value of time. (Recall that time simply scales $x$ and $r$.) Figure 4 shows comparisons of the flow as given by solutions of the Euler equations and the leading shock front as determined by shock dynamics for three initial shock angles. The initial Mach number of the shock is $M_{\mathrm{s}}=6.34$ so that the area-Mach number rule in the strong shock limit is used. The Euler solution is displayed as a pseudo-schlieren image while the shock dynamics solution is given by the solid white curve. As may be seen, the shock shapes from shock dynamics agree very well with those given by the Euler equations. Also, the location of the shock-shock agrees very well with that of the triple point of the Mach reflections of the Euler computation, except in the case of $\beta=20^{\circ}$, where the shock-shock is ahead of the triple point. This is a scale effect, also observed in the self-similarity test in Hornung (2000), that results from the finite grid size in the numerics. This causes the Mach stem to appear only at a finite time since it is too small to be resolved by the grid initially. This scale effect is less pronounced at the larger shock angles, where the scale of the Mach reflection is larger. As mentioned previously, shock dynamics always predicts a planar Mach disk in contrast to the detailed Mach reflection observed here in the solution of the Euler equations resulting from features that form behind the leading shock and overtake it.

A comparison of shock positions for a range of initial shock Mach numbers, 


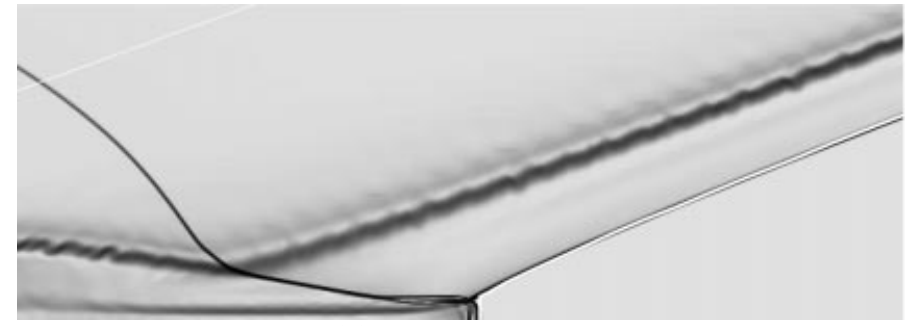

(a) $t=3.19, \beta=20^{\circ}$

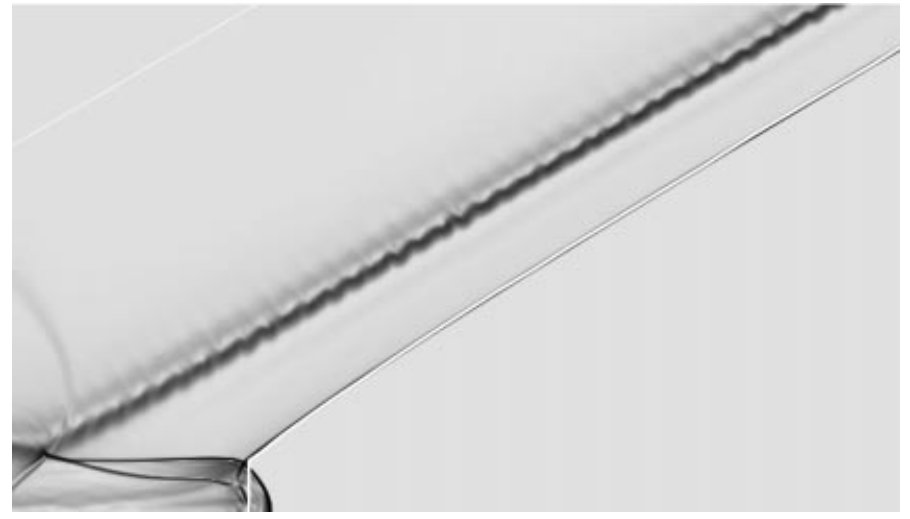

(b) $t=3.60, \beta=30^{\circ}$

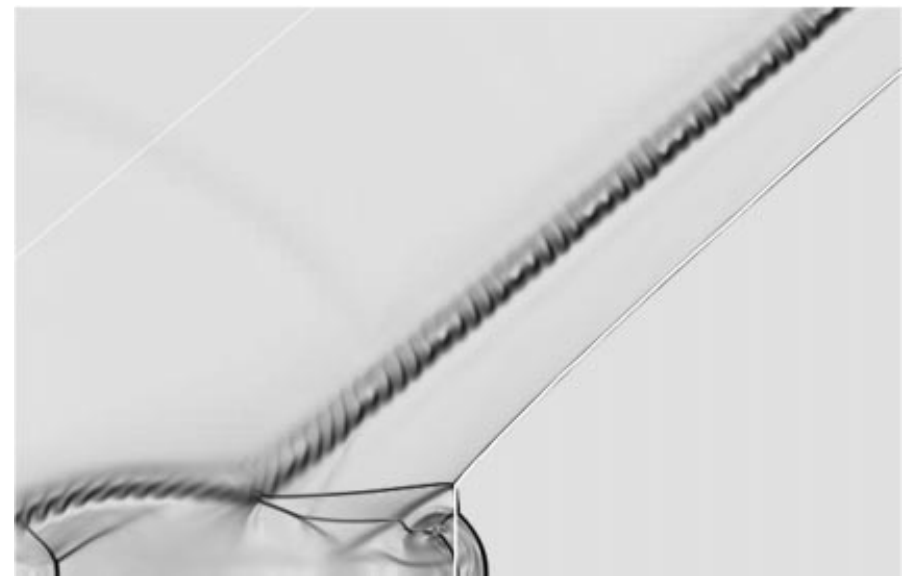

(c) $t=4.28, \beta=40^{\circ}$

FIGURE 4. Comparison of numerical solutions with shock dynamics results. The Euler solutions are portrayed as pseudo-schlieren images, in which the greyshading is a monotonic function of the magnitude of the fractional density gradient. The location of the shock wave according to shock dynamics is shown as a white curve with a slope discontinuity at the shock-shock. The straight white line in the top left corner of each frame is the original location of the shock. $M_{\mathrm{s}}=6.34$, $\gamma=1.4$.

including weak shocks, is given in figure 5. For this comparison, we solve the ODEs in (4.4) and (4.5), and the boundary conditions in (4.6) with the full area-Mach number rule (cf. Whitham 1974, equation 8.37). The particular values of $M_{\mathrm{s}}$ used in this comparison are chosen to correspond to the ones given in Hornung (2000) for the initial angle $\beta=40^{\circ}$ and $\gamma=1.4$. It may be seen that shock dynamics again 
gives excellent agreement with the shock shape from the Euler computation, but the location of the Mach stem from shock dynamics is noticeably ahead of that from the Euler computations in the case of the very weak shocks. The reason for the increasing discrepancy between the shock-shock and triple-point locations as the Mach number decreases is due to the fact that the approximate theory of shock dynamics becomes inaccurate for very weak shocks.

\section{Relation to the Guderley singularity}

We interpret the steepening of the shock with decreasing radius in the oblique implosion as being analogous to the speed increase with decreasing radius of the cylindrical implosion. In the latter, the shock location is given, according to the Guderley theory, by

$$
r=C_{0}\left(t_{0}-t\right)^{m_{0}}
$$

where $t_{0}$ is the time at which the shock hits the axis, $r$ is the radial distance of the shock from the axis, $C_{0}$ is a constant, and $m_{0}=0.835217$ for a perfect gas with $\gamma=1.4$.

If the oblique implosion has any relation to the Guderley singularity, we might guess that the shape of the shock near the axis would be of the form

$$
r=C\left(x-x_{0}\right)^{m},
$$

where $x_{0}$ is the location at which the shock would intersect the axis if there were no Mach reflection, and $C$ is a constant. In the far field, the shock has to asymptote to the position that a plane shock would have reached in the same elapsed time. A suitable curve that has both of these properties is the generalized hyperbola

$$
\left(\frac{x}{a}\right)^{1 / m}-\left(\frac{r}{b}\right)^{1 / m}=\left(\frac{x_{0}}{a}\right)^{1 / m} .
$$

For fitting such a curve to the computed shock shape, it turns out that we have very limited freedom. The slope of the asymptote determines $a / b$, the location of the asymptote is determined by the position a plane shock would have reached in the same elapsed time, and $a$ and $x_{0}$ are determined by matching two points of the computed shock with the hyperbola, one of which is effectively fixed by the location of the asymptote. The only remaining fitting parameter is therefore the exponent $m$. The quality of the resulting fits may be seen in figure 6 showing superpositions of the curve in white and the computed pseudo-schlieren image for the case of $M_{\mathrm{s}}=6.34, \gamma=1.4$.

A plot of the resulting values of $m$ against the initial shock angle is shown in figure 7. Here, the point $(\beta, m)=\left(0^{\circ}, m_{0}\right)$ of the Guderley solution has been added, and a further point $\left(90^{\circ}, 0.5\right)$ has been included on the basis of the argument that the solution at $\beta=90^{\circ}$ has to be regular. The error of determining $m$ from the fit is approximately equal to the vertical extent of the plotted symbols. The points fit quite well onto a parabola of best fit to them. This result adds to the confidence with which one can conclude that the steepening of the shock is intimately connected with the Guderley theory.

\section{The steady-flow analogue}

Mölder et al. (1997) presented experimental and computational results that exhibit shock steepening and Mach reflection when an oblique shock approaches its axis of 


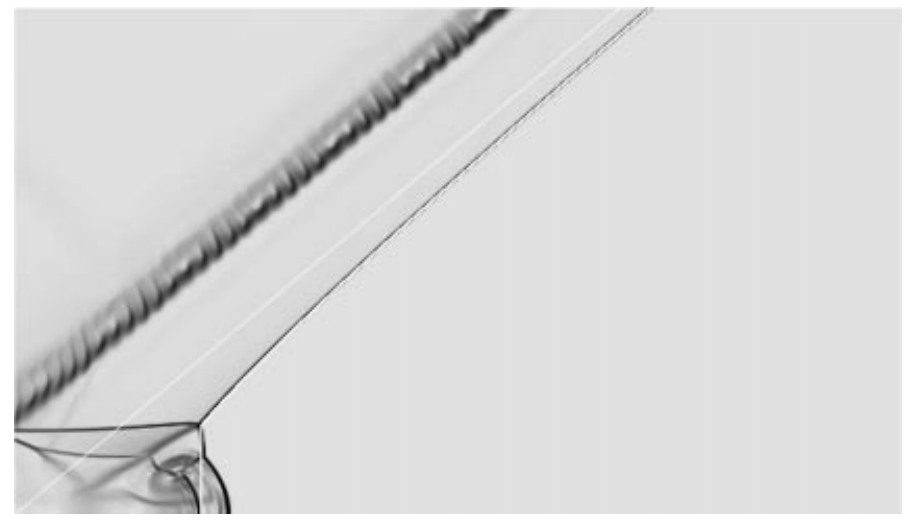

(a) $M_{\mathrm{s}}=4.52$

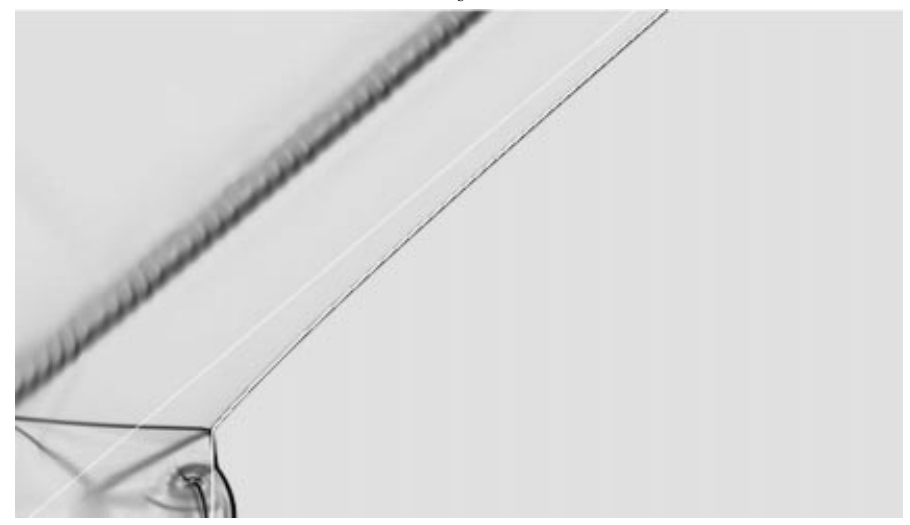

(b) $M_{\mathrm{s}}=2.93$

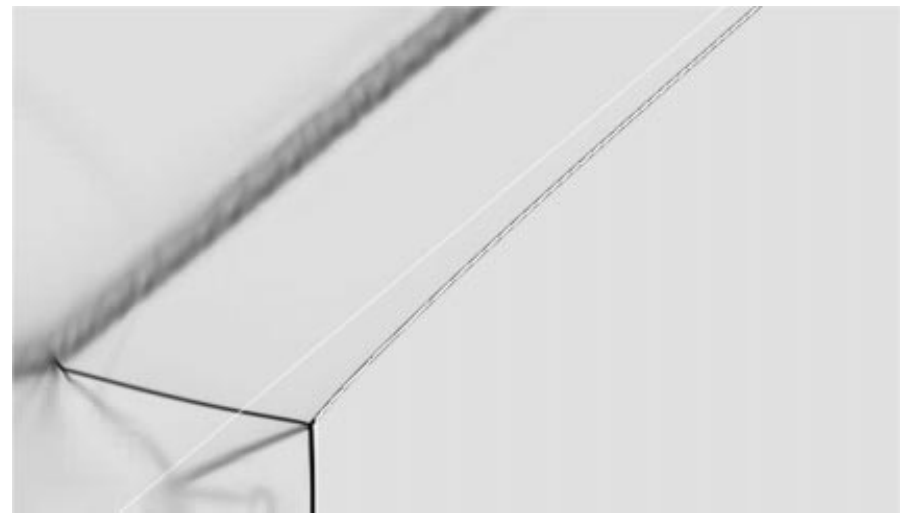

(c) $M_{\mathrm{s}}=2.15$

FIGURE $5(a-c)$. For caption see facing page.

symmetry in the case of steady flow. They used an extension of the Taylor-Maccoll theory, for steady axisymmetric supersonic flow over cones, to shock angles greater than $90^{\circ}$, in order to design ring-shaped bodies that would generate conical shocks. Their observations may be summarized by saying that they could not generate conical shock waves in this manner, and that the shock always steepened and formed a simple Mach reflection at the axis. Even in the case of an extremely weak shock generated in this manner, for which the experiment could not resolve the Mach stem or the 


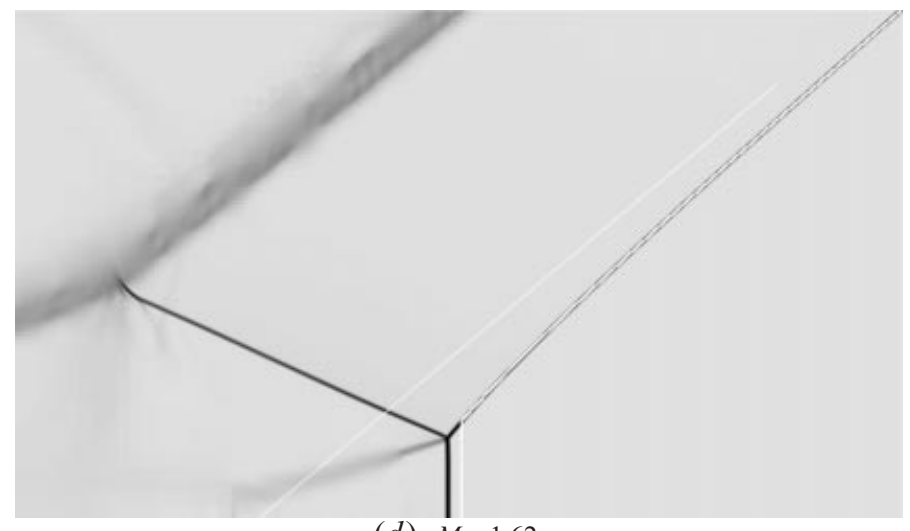

(d) $M_{\mathrm{s}}=1.62$

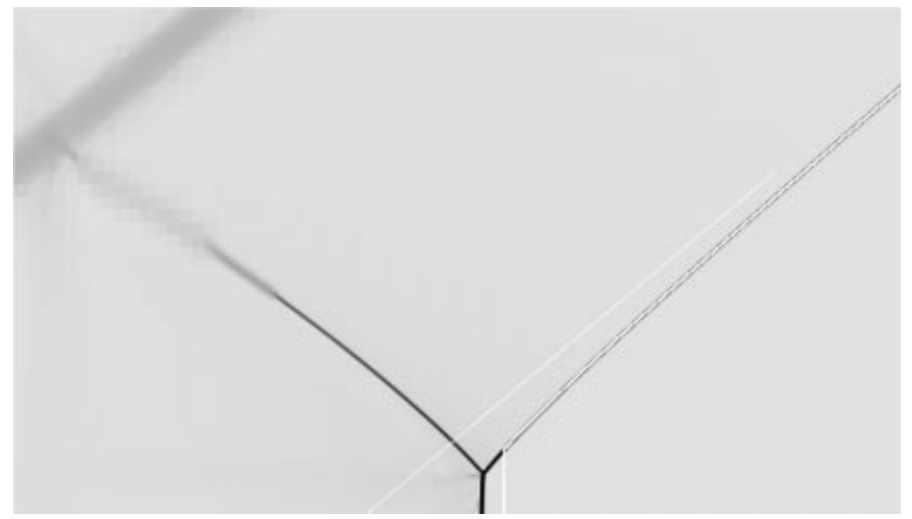

(e) $M_{\mathrm{s}}=1.19$

FIGURE 5. Comparison of numerical solutions with shock dynamics results for weaker shocks at $\beta=40^{\circ}, \gamma=1.4$. The straight white line is the location to which a plane shock would have propagated in the same time. It is therefore the far-field asymptote of the curved shock.

steepening, computation of the flow with very high resolution indicated that both were present on a very small scale.

We consider this phenomenon to be a steady-flow analogue of the type of reflection studied here. From the viewpoint of an observer travelling with the triple point of the oblique implosion, the flow is very similar to that of the steady, inward-facing shock.

The approximate equivalence of the steady and the self-similar unsteady flows suggests that the three different shock reflection structures found by Hornung (2000) also occur in the steady case. In order to investigate this further, some computations of the flow field of an overexpanded jet were made. As in Hornung (2000), these computations were made with the computational system Amrita by Quirk (1998) applied to the Euler equations with adaptive mesh refinement. Three such flows are shown in figure 8. As may be seen, here too the shock steepens and a Mach reflection is formed at the axis. The steepening occurs despite the fact that the streamlines in this flow have curvature of opposite sign to that of the shock. This is true also in the cases studied by Mölder et al. (1997).

Also, the three cases show that, while a reflection configuration similar to Hornung's type V reflection does occur, see figure $8(c)$, type DV could not be found. Simple Mach reflection is the type that occurs at the conditions where type DV is observed in the self-similar unsteady flows, see figures $8(a)$ and $8(b)$. The jet calculations were 
(a)

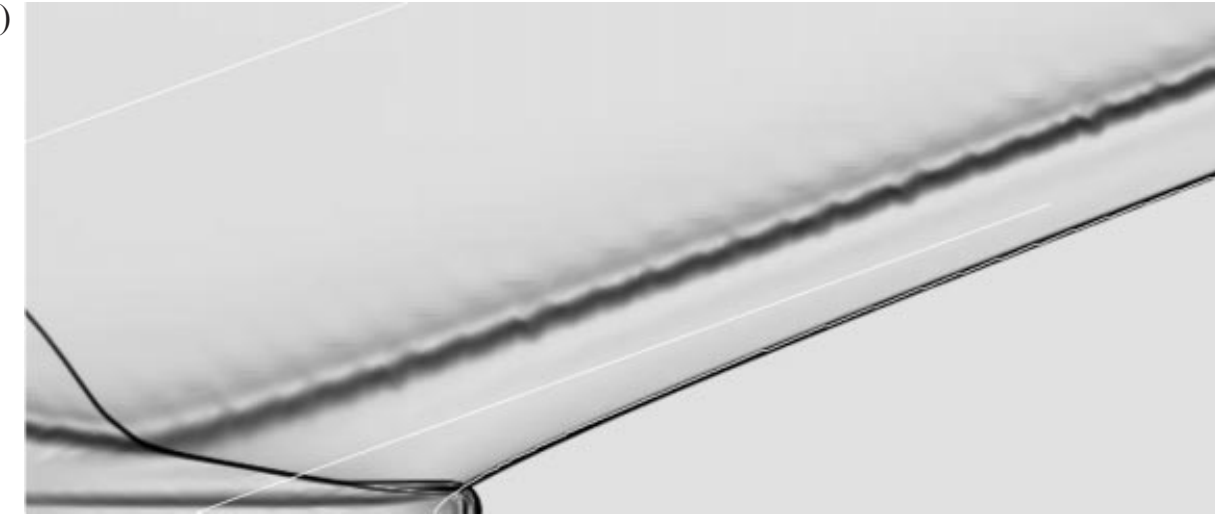

(b)

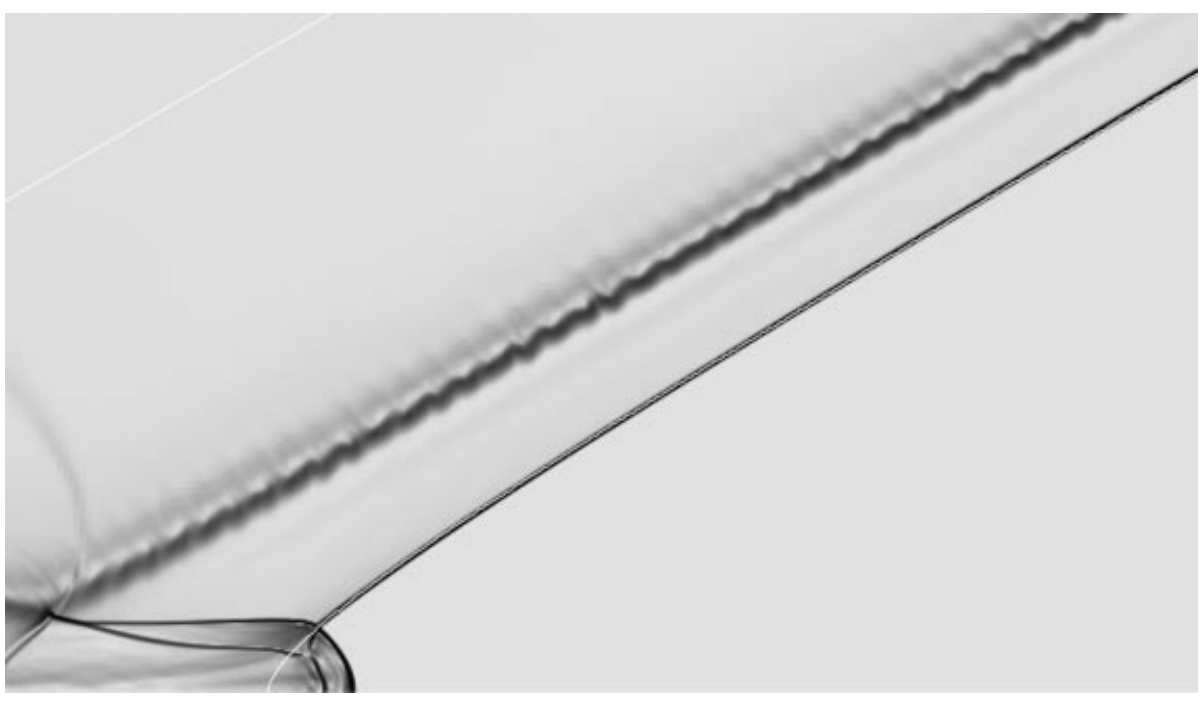

(c)

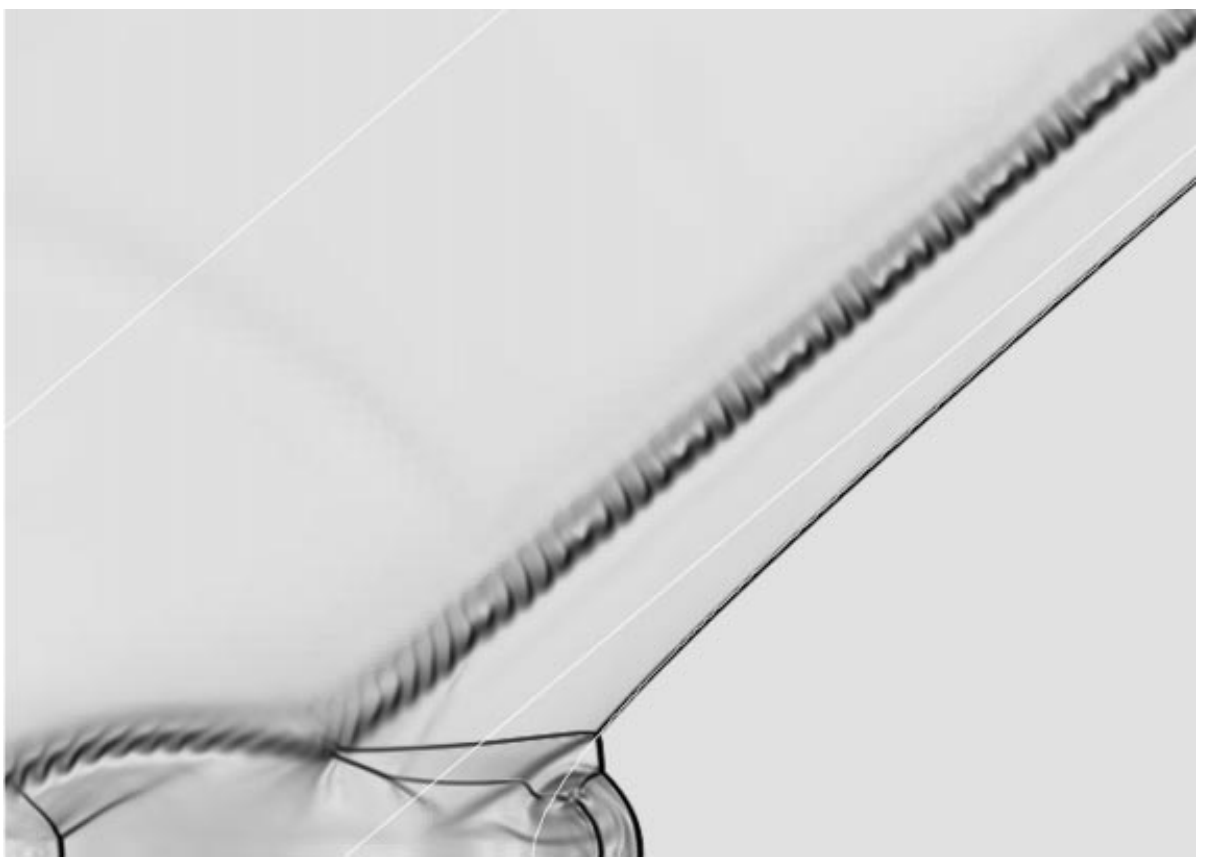

FiguRE $6(a-c)$. For caption see facing page. 
(d)

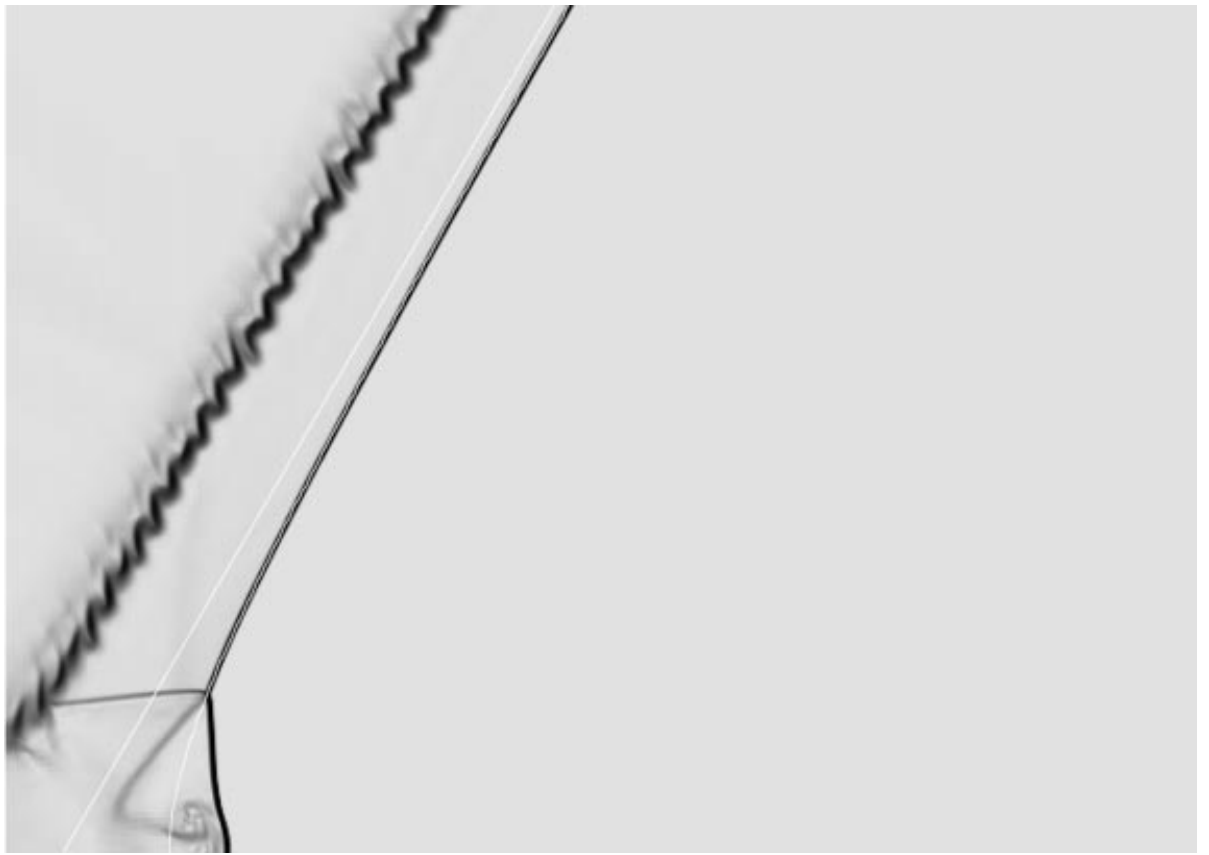

FIGURE 6. Fit of a generalized hyperbola to the computed shock shape for $\beta=20^{\circ}, 30^{\circ}, 40^{\circ}$, and $60^{\circ}$. The two straight white lines show the original location of the diaphragm and the asymptote of the hyperbola, which was calculated as the position a plane shock would have reached in the same elapsed time as in the computation of the axisymmetric flow (the latter is missing in the $\beta=30^{\circ}$ case). The white curve, which falls almost exactly on the black shock of the computation, is the hyperbola of best fit.

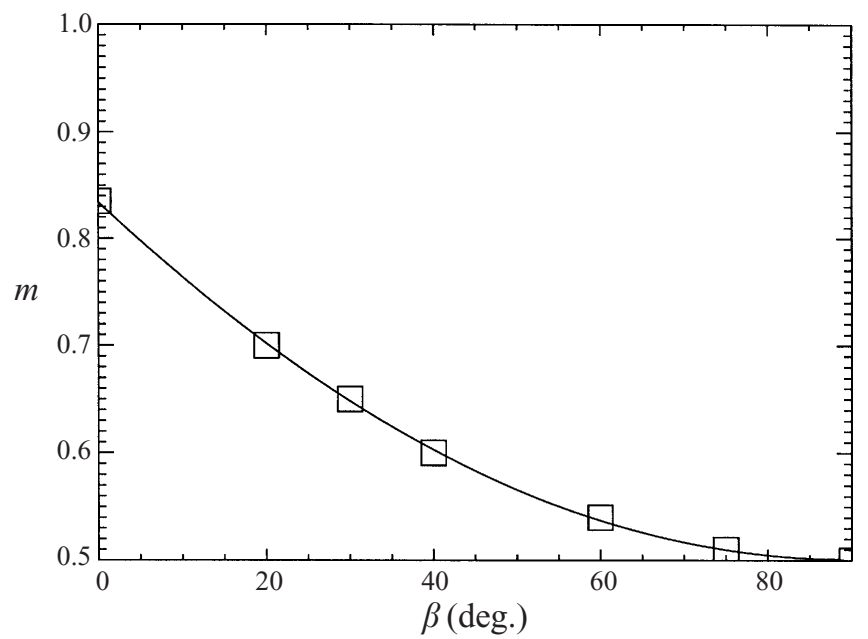

Figure 7. Plot of the values of $m$. At $0^{\circ}$, the Guderley value $(0.8352)$ applies. At $90^{\circ}$ the shock shape must be regular. The value $m=0.5$ gives such a regular curve, so that one might speculate that this is the right value for the normal shock. The curve is a least-squares fit of a parabola to the five points obtained from fits like those shown in figure 6 and the two end points. The error in these values, estimated from where the quality of fit deteriorates noticeably, is approximately equal to the size of the symbols. 


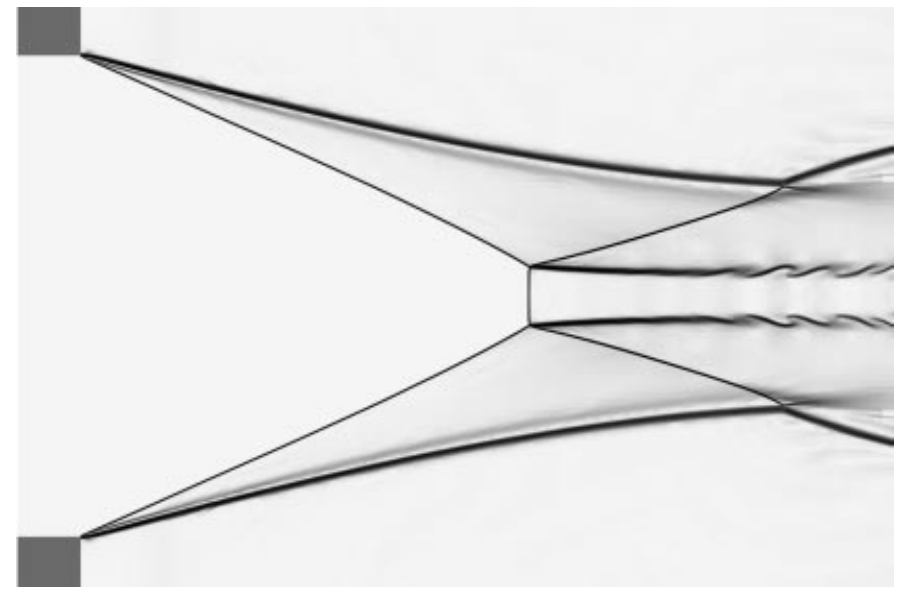

(a) $M_{\infty}=6.0, p_{b} / p_{j}=6.6, \gamma=1.4$

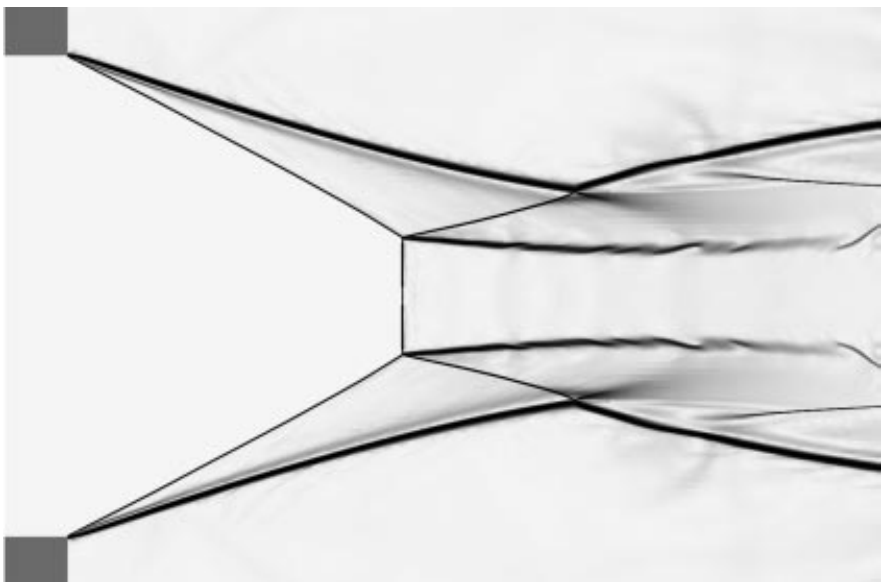

(b) $M_{\infty}=6.5, p_{b} / p_{j}=9.6, \gamma=1.4$

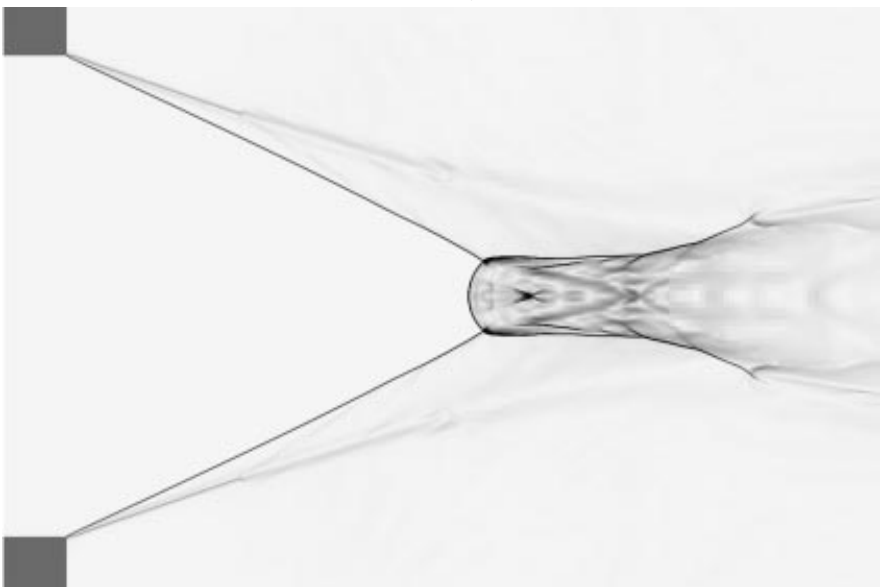

(c) $M_{\infty}=6.0, p_{b} / p_{j}=6.6, \gamma=1.1$

FIGURE 8. Shock formed at the lip of an axisymmetric overexpanded jet. Note that the shock steepens, although the streamline coming from the jet lip curves concave outward. The symbols $p_{b}$ and $p_{j}$ denote the back pressure and jet pressure respectively. Conditions for the frames correspond to conditions where types $(a) \mathrm{S},(b) \mathrm{DV}$ and $(c) \mathrm{V}$ occur in the self-similar unsteady flows. The type DV interaction could not be observed, but $(c)$ is similar in some ways to type V. 
performed with the following initial conditions: uniform flow at $M_{\infty}$ within the region of radius equal to the jet exit radius, and stationary gas at higher pressure outside this radius. At the conditions where the unsteady flows exhibit type DV, this form of the reflection does, in fact, occur in the early, unsteady phase of the evolving flow. However, the vortex ring of those configurations weakens, and disappears altogether by the time a steady state is reached (figure $8 b$ ) so that the reflection configuration ends up as type $\mathrm{S}$.

\section{Conclusions}

The flow that occurs when an initially conical shock of initially uniform strength reflects from its axis of symmetry was considered. The shock steepens as it approaches the axis, and three different Mach reflection structures occur near the axis. The selfsimilar motion of the leading shock front as described by the theory of geometrical shock dynamics was compared to shock waves determined by numerical solutions of the Euler equations for the entire flow. Very good agreement was found for the shock shape for both strong and weak shocks, and in the triple-point location of the Mach reflection for strong shocks. This flow is intimately related to the Guderley singularity, and quantitative fits of the computed steepening shock waves to a generalized hyperbola, suggested by analogy with the Guderley flow, showed interesting similarities. Shock steepening occurs also in the steady flow analogue of the oblique implosion problem, exemplified here by overexpanded supersonic jets.

We wish to thank Dale Pullin and Gerry Whitham for valuable discussions.

\section{REFERENCES}

Bryson, A. E. \& Gross, R. W. F. 1961 Diffraction of strong shocks by cones cylinders and spheres. J. Fluid Mech. 10, 1-16.

Hornung, H. G. 2000 Oblique shock reflection from an axis of symmetry. J. Fluid Mech. 409, 1-12.

Mölder, S., Gulamhussein, A., Timofeev, E. \& Voinovich, P. 1997 Focusing of conical shocks at the center-line of symmetry. In Shock Waves (ed. A. F. P. Houwing \& A. Paull), pp. 875-880. Panther Publishing and Printing, Canberra.

Quirk, J. J. 1998 Amrita-a computational facility (for cfd modelling). VKI 29th CFD Lecture Series, ISSN 0377-8312.

RYLov, A. I. 1990 On the impossibility of regular reflection of a steady-state shock wave from the axis of symmetry. Prikl. Mat. Mech. 54, 200-203.

Schwendeman, D. W. \& Whitham, G. B. 1987 On converging shock waves. Proc. R. Soc. Lond. A 413, 297-311.

SCHWEndemAn, D. W. 1993 A new numerical method for shock-wave propagation based on geometrical shock dynamics. Proc. R. Soc. Lond. A 441, 331-341.

Takayama, K., Kleine, H. \& GRönig, H. 1987 An experimental investigation of the stability of converging cylindrical shock waves in air. Exps. Fluids 5, 325-322.

Whitham, G. B. 1959 A new approach to problems of shock dynamics. Part 2. Three-dimensional problems. J. Fluid Mech. 5, 369-386.

Whitнaм, G. B. 1974 Linear and Nonlinear Waves. Wiley. 